\title{
Efeito do extrato de Bidens pilosa L., Mel e pomadas homeopática e alopática na cicatrização de feridas cutâneas de ratos Wistar
}

\author{
[Effect of Bidens pilosa L., Honey extract and homeopathic and allopathic ointments on the healing \\ of skin wounds of Wistar rats] \\ C.E.C. Santos ${ }^{1}$, M.G.S. Carvalho ${ }^{1}$, B.E. Costa ${ }^{1}$, W.G. Ferreira Júnior ${ }^{2}$, C.C. Lima ${ }^{3}$, \\ P.P. Corsetti ${ }^{3}$, J.A.D. Garcia ${ }^{2,3 *}$ \\ ${ }^{1}$ Aluno de pós-graduação - Universidade José do Rosário Vellano - Alfenas, MG \\ ${ }^{2}$ Instituto Federal de Educação Ciência e Tecnologia do Sul de Minas Gerais - Machado, MG \\ ${ }^{3}$ Universidade José do Rosário Vellano - Alfenas, MG
}

\begin{abstract}
RESUMO
Cicatrização de ferida é um processo dinâmico, que tem por objetivo restaurar a continuidade do tecido lesionado. No entanto, em alguns casos, é necessário favorecer condições adequadas para viabilizar o processo fisiológico. Neste estudo foram utilizados ratos Wistar, divididos aleatoriamente entre cinco grupos, com 12 animais cada, sendo eles: grupo $\mathrm{P}$ (Bidens pilosa L.), grupo mel, grupo Co1 (pomada comercial alopática), grupo Co2 (pomada comercial homeopática) e grupo CT (controle). As lesões foram geradas por incisão com punch de $8 \mathrm{~mm}$, sendo tratadas diariamente de forma tópica. Foram eutanasiados quatro animais por grupo, no terceiro, sétimo e $14^{\circ}$ dias do experimento, e o material coletado foi armazenado em formalina $10 \%$ e encaminhado para processamento histológico. Posteriormente, realizouse a contagem de leucócitos mononucleares, fibroblastos e neovasos e avaliou-se a arquitetura de fibras colágenas. Os resultados da contagem foram analisados pela ANOVA, seguida pelo teste de Tukey $(\mathrm{P}<0,05)$. O modelo experimental proposto neste estudo demonstrou que todos os tratamentos apresentaram potencial cicatrizante, com exceção do mel. A aplicação tópica do creme do extrato de Bidens pilosa L. a $10 \%$ apresentou melhor perfil anti-inflamatório; a pomada alopática apresentou boa aderência à superfície da lesão e a pomada homeopática, grande potencial angiogênico, com menor tempo de cicatrização.
\end{abstract}

Palavras-chave: cicatrização de feridas, colágeno, fibroblastos, fitoterápico, homeopatia.

\begin{abstract}
Wound healing is a dynamic process that aims to restore the continuity of injured tissue. However, in some cases it is necessary to favor adequate conditions to enable the physiological process. Wistar rats were randomly divided into 5 groups with 12 animals each, namely: group P (Bidens pilosa L.), group honey, group Co1 (commercial allopathic ointment), group Co2 (commercial homeopathic ointment) and group $C T$ (control). The lesions were generated by an $8 \mathrm{~mm}$ punch incision and were treated topically daily. Four animals per group were euthanized on the $3 \mathrm{rd}$, 7th and 14th day of the experiment and the collected material was stored in 10\% formalin and sent for histological processing, after which mononuclear, fibroblasts and neovascular leukocytes were counted and collagen fiber architecture was evaluated. Counting results were analyzed by ANOVA, followed by Tukey test $(p<0.05)$. The experimental model proposed in this study showed that all treatments had healing potential, except honey. The topical application of 10\% Bidens pilosa L. extract cream showed the best anti-inflammatory profile; Allopathic ointment showed good adhesion to the surface of the lesion and homeopathic ointment showed great angiogenic potential with shorter healing time.
\end{abstract}

Keywords: wound healing, collagen, fibroblasts, herbal medicine

Recebido em 24 de julho de 2019

Aceito em 12 de fevereiro de 2020

*Autor para correspondência (corresponding author)

E-mail: jadiasgarcia@gmail.com 


\section{INTRODUÇÃO}

As feridas cutâneas podem ser causadas por traumas ou procedimentos cirúrgicos. Após a ocorrência da lesão cutânea, inicia-se o processo de reparo, a qual é dividia em três fases: inflamatória, caracterizada pela migração de macrófagos para a área lesionada, que, por sua vez, secretam fatores de crescimento, citocinas pró-inflamatórias e anti-inflamatórias e fagocitam microrganismos; fase proliferativa, determinada pela proliferação de novos vasos sanguíneos; e fase de remodelamento ou maturação, quando ocorre a deposição de colágeno de maneira organizada. O processo de cicatrização é sistêmico e dinâmico, e as fases que o compõem ocorrem de maneira interligada, tendo por objetivo restaurar a continuidade do tecido lesionado (Oliveira e Dias, 2012).

No entanto, em alguns casos, é necessário favorecer condições adequadas para viabilizar o processo fisiológico da cicatrização, e consequentemente muito investimento tem sido realizado na compreensão da dinâmica cicatricial, nas pesquisas e no desenvolvimento de técnicas e recursos para o tratamento das feridas cutâneas em humanos e animais (Mandelbaum et al., 2003), buscando por produtos que favoreçam uma reparação tecidual de estrutura e função semelhante aos da pele íntegra em menor tempo possível (Araújo, 2016).

As plantas medicinais e os produtos naturais são utilizados desde a antiguidade para o tratamento de diversas doenças e têm se destacado no contexto de reparação tecidual por apresentarem alto poder antibacteriano e anti-inflamatório, boa nutrição ao tecido e baixo custo (Moreski et al, 2018). Produtos derivados de plantas e associações são comercializados para o tratamento de feridas; um exemplo é a alantoína, extraída da planta Symphytum officinale, conhecida como "confrei", que se destaca pelo potencial hidratante, removedor de tecido necrótico e cicatrizante (Araújo et al., 2010). A associação obtida das plantas Bellis perenis, Calendula officinalis e Myristica sebifera também apresentou perfil cicatrizante, pois diminuiu o tempo de cicatrização e o tamanho das feridas induzidas em ratos Wistar (Fernandes et al., 2014). Outro produto muito utilizado no tratamento de diversas doenças, incluindo a cicatrização de feridas (Marques et al., 2015), é o mel, um produto natural, derivado da coleta do néctar das flores pelas abelhas, rico em nutrientes (Alves et al., 2005).

As plantas medicinais vêm sendo alvo de experimentações, a fim de se buscar respaldo científico e segurança para o seu uso. Uma das plantas medicinais utilizadas na medicina popular e que vêm sendo fonte de pesquisas é a Bidens pilosa L., pertencente à família Asteracea. Algumas pesquisas têm demonstrado o potencial dessa planta antimalárico (Oliveira et al., 2004), antimicrobiano (Haida et al., 2007), antifúngico (Tagami et al., 2009), analgésico e antiinflamatório (Fotso et al., 2014). Esses potenciais medicinais se devem a compostos encontrados na planta, como poliacetilenos, flavonoides, alcaloides, esteroides, taninos, ácido linoleico e ácido linolênico (Geissberger e Séquin, 1991).

Em razão da busca constante por produtos eficazes no processo de cicatrização e do pouco conhecimento do potencial cicatrizante dessa planta, o presente trabalho teve como objetivo comparar o efeito do creme do extrato de Bidens pilosa L. a $10 \%$, do mel e das pomadas comerciais alopática e homeopática no tratamento de feridas cutâneas, em ratos da linhagem Wistar (Rattus norvegicus).

\section{MATERIAL E MÉTODOS}

O experimento foi conduzido no Laboratório de Pesquisa da Universidade José do Rosário Vellano (Unifenas), em Alfenas. Utilizaram-se 60 ratos (Rattus norvegicus), Wistar, brancos,

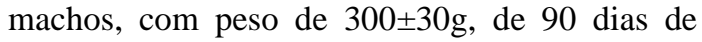
idade, obtidos do Biotério Central da Unifenas, Campus Alfenas. O protocolo experimental foi aprovado pelo Comitê de Ética em Pesquisa, Parecer N. 05A/ 2016.

As partes aéreas (folhas e caules) de B. pilosa foram coletadas na Trilha Ecológica da Biologia, localizada no Instituto Federal de Educação, Ciência e Tecnologia do Sul de Minas Gerais (IFSULDEMINAS, Campus Machado), no município de Machado (21\%41'58"S 4553'18'W). O material botânico coletado foi identificado e herborizado no Herbário do IFSULDEMINAS, Campus Machado. A obtenção do extrato aquoso de $B$. pilosa foi dada por infusão, utilizando-se um béquer contendo $5 \mathrm{~g}$ de folhas frescas e $50 \mathrm{~mL}$ de água destilada fervente. 
Em seguida, o béquer foi tampado com placa de Petri, para a obtenção do extrato aquoso na concentração de $10 \%$, e, depois de cinco minutos, o extrato foi filtrado (Bach e Silva, 2010). Após a obtenção do extrato aquoso, este foi filtrado e evaporado em pressão reduzida e posteriormente liofilizado, obtendo-se o extrato seco (Hernandes et al., 2010). Para preparação do creme a $10 \%$, misturaram-se $6 \mathrm{~mL}$ do extrato aquoso a $60 \mathrm{~g}$ do creme base, manipulado na Farmácia de Manipulação da Unifenas e conservado em temperatura ambiente de $22^{\circ} \mathrm{C}$ (Coelho et al., 2010).

O mel foi coletado em colmeias de abelhas africanizadas (Apis mellifera), no setor de Apicultura do IFSULDEMINAS, Campus Machado, e mantido em temperatura ambiente para evitar a sua cristalização. As pomadas utilizadas no experimento foram alopática (alantoína e óxido de zinco, Alantol ${ }^{\circledR}$, Vetnil, Brasil) e homeopática (Bellis perenis + Calendula officinalis + Myristica sebifera, $\mathrm{CMR}^{\circledR}$, Real H, Brasil).

Os animais foram submetidos à anestesia com Thiopentax $^{\circledR}$ (tiopental sódico, Cristália, Brasil) $(10 \mathrm{mg} / \mathrm{kg})$ intraperitoneal (IP) e à depilação manual na região dorsocervical; as feridas foram induzidas por uma incisão circular da pele por meio de punch de $8 \mathrm{~mm}$. Após a incisão, os animais receberam, por via subcutânea (SC), uma única dose de dipirona $(10 \mathrm{mg} / \mathrm{kg})$. Os animais foram divididos aleatoriamente em cinco grupos experimentais, e os tratamentos tópicos das feridas foram realizados após higienização com água e degermante neutro diariamente, durante 14 dias: grupo controle $(\mathrm{CT}, \mathrm{n}=12)$ : ferida higienizada com água e degermante neutro; grupo mel (Mel, $\mathrm{n}=12)$ : ferida tratada com mel in natura; grupo picão $(\mathrm{P}, \mathrm{n}=12)$ : ferida tratada com creme de extrato de Bidens pilosa L. a 10\%; grupo pomada comercial alopática $(\mathrm{Co} 1, \mathrm{n}=12)$; grupo pomada comercial homeopática $(\mathrm{Co} 2, \mathrm{n}=12)$. Os ratos foram mantidos em caixas de polietileno, separadamente, para evitar traumas por outros animais, pois as feridas não receberam bandagens, e as soluções do tratamento do dia anterior eram removidas com a higienização diária. Todos os animais receberam ração comercial (Nuvital ${ }^{\circledR}$, Brasil) e água filtrada ad libitum. No terceiro, sétimo e $14^{\circ}$ dias de tratamento, foram eutanasiados quatro animais de cada grupo com dose letal de tiopental sódico $(100 \mathrm{mg} / \mathrm{kg})$ intraperitoneal (IP).

Após a eutanásia, foram coletadas amostras do tecido em processo de cicatrização, abrangendo uma margem de $4 \mathrm{~mm}$ de segurança. O material coletado no terceiro, sétimo e $14^{\circ}$ dias foi fixado em formalina a $10 \%$, por $48 \mathrm{~h}$, e incluído em parafina para cortes histológicos de $5 \mu \mathrm{m}$ (Junqueira e Carneiro, 2004). Para análise morfológica geral e contagem de células mononucleares, de neovasos e de fibroblastos, as lâminas receberam a coloração hematoxilinaeosina (HE), e, para visualização da densidade das fibras colágenas, as lâminas foram coradas com picro sirius red. Os cortes histológicos em $\mathrm{HE}$ e picro sirius red foram escaneados pelo scanner Pannoramic DESK, da 3D HISTECH, com aumento de 400x, e posteriormente analisados por meio do programa PannoramicViewer.

Oito campos de cada corte histológico por animal passaram por análises em que a média do número de células mononucleares, de neovasos e de fibroblastos foi calculada. O resultado dessa contagem foi avaliado estatisticamente pela ANOVA e comparado por meio do teste de Tukey, com significância $\mathrm{P}<0,05$. Os resultados foram expressos pela média \pm erro-padrão da média (média \pm EPM).

\section{RESULTADOS}

Durante o experimento, os animais ganharam peso e não houve diferenças significativas entre os grupos. Quanto ao consumo de dieta líquida e sólida, não houve diferença significativa entre os grupos e ele foi suficiente para fornecer hidratação e energia necessária para manter as atividades corporais diárias. $\mathrm{Na}$ avaliação clínica das feridas cutâneas (Fig. 1), os animais do grupo controle (CT) apresentaram acúmulo moderado de crostas sobre a lesão e obtiveram cicatrização completa no $14^{\circ}$ dia de tratamento, com boa aparência estética. 


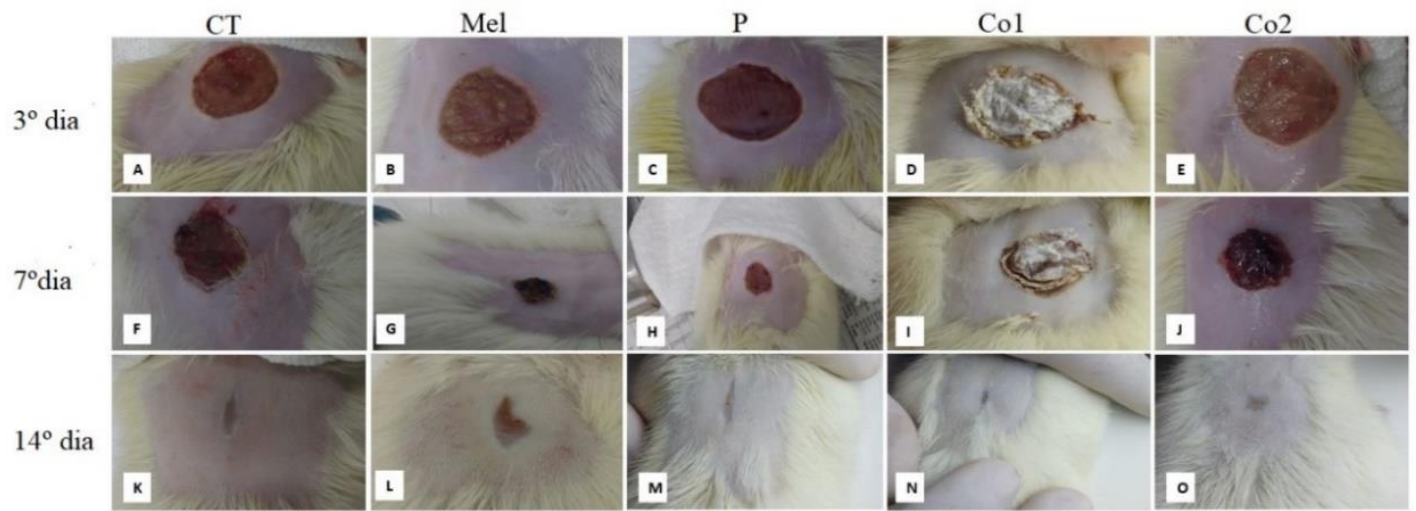

Figura 1. Imagem demonstrativa da evolução clínica das feridas cutâneas de ratos Wistar (Rattus norvegicus), ao terceiro dia (A-E), sétimo dia (F-J) e $14^{\circ}$ dia (K-O) de experimento dos grupos controle (CT), mel, creme do extrato de Bidens pilosa L. a 10\% (P), pomada comercial alopática - alantoína e óxido de zinco (Co1), pomada comercial homeopática - Bellis perenis + Calendula officinalis + Myristica sebifera $(\mathrm{Co} 2)$.

O grupo mel caracterizou-se por uma boa evolução inicial, sendo visível a contração das bordas da ferida até o sétimo dia de tratamento, porém houve acúmulo significativo de crostas e não ocorreu boa cicatrização ao final do experimento. O grupo que recebeu o creme a $10 \%$ do extrato de $B$. pilosa $(\mathrm{P})$ destacou-se por não haver acúmulo excessivo de crostas e, apesar de a ferida demonstrar cicatrização lenta nos primeiros dias, do sétimo dia em diante houve boa contração cicatricial, obtendo-se o fechamento completo da lesão no $12^{\circ}$ dia de tratamento, com boa aparência estética. O grupo dos animais tratados com a pomada alopática (Co1) destacou-se pela aderência à superfície da lesão e pela predisposição a uma formação excessiva de crostas, contudo houve cicatrização completa com 13 dias de tratamento, com boa aparência estética.
O grupo tratado com a pomada homeopática (Co2) apresentou boa resposta cicatricial desde o início do tratamento, não houve predisposição ao acúmulo excessivo de crostas e ocorreu a cicatrização por completo no $11^{\circ}$ dia de tratamento, com boa aparência estética.

$\mathrm{Na}$ análise dos cortes histológicos das feridas cutâneas dos ratos, após três dias de tratamento, observou-se aumento significativo do número de fibroblastos nos animais dos grupos tratados com a pomada $\mathrm{Co} 2$ e com mel, quando comparados aos demais grupos estudados (Tab. 1 e Fig. 2). Os resultados foram expressos em médiaterro-

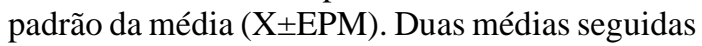
pela mesma letra não são diferentes entre si $(\mathrm{P}>0,05)$ ao teste de Tukey.

Tabela 1. Média do número de fibroblastos, das células mononucleares e dos neovasos por campo das secções histológicas das feridas cutâneas de ratos Wistar (Rattus norvegicus), no terceiro, sétimo e $14^{\circ}$ dias de experimento dos grupos controle (CT), mel, creme do extrato de Bidens pilosa L. a 10\% (P), pomada comercial alopática - alantoína e óxido de zinco (Co1), pomada comercial homeopática - Bellis perenis + Calendula officinalis + Myristica sebifera $(\mathrm{Co} 2)$

\begin{tabular}{lllllll}
\hline & & \multicolumn{1}{c}{ CT } & \multicolumn{1}{c}{ Mel } & \multicolumn{1}{c}{ P } & \multicolumn{1}{c}{ Co1 } & \multicolumn{1}{c}{ Co2 } \\
\hline \multirow{3}{*}{$3^{\mathrm{o}}$ dia } & Fibroblastos/campo & $50 \pm 3^{\mathrm{b}}$ & $70 \pm 12^{\mathrm{a}, \mathrm{b}}$ & $50 \pm 3^{\mathrm{b}}$ & $40 \pm 7^{\mathrm{b}}$ & $90 \pm 12^{\mathrm{a}}$ \\
& Células mononucleares/campo & $40 \pm 3^{\mathrm{a}, \mathrm{b}}$ & $30 \pm 5^{\mathrm{b}}$ & $10 \pm 1^{\mathrm{c}}$ & $60 \pm 10^{\mathrm{a}}$ & $40 \pm 8^{\mathrm{a}, \mathrm{b}}$ \\
& Neovasos/campo & $30 \pm 6^{\mathrm{b}}$ & $40 \pm 6^{\mathrm{b}}$ & $50 \pm 6^{\mathrm{a}, \mathrm{b}}$ & $30 \pm 3^{\mathrm{b}}$ & $60 \pm 9^{\mathrm{a}}$ \\
\multirow{2}{*}{$7^{\mathrm{o}} \mathrm{dia}$} & $50 \pm 6^{\mathrm{a}, \mathrm{b}}$ & $70 \pm 7^{\mathrm{a}, \mathrm{b}}$ & $50 \pm 5^{\mathrm{b}}$ & $60 \pm 3^{\mathrm{a}, \mathrm{b}}$ & $80 \pm 10^{\mathrm{a}}$ \\
& Fibroblastos/campo & $30 \pm 3^{\mathrm{a}}$ & $30 \pm 6^{\mathrm{a}}$ & $10 \pm 3^{\mathrm{b}}$ & $30 \pm 6^{\mathrm{a}}$ & $30 \pm 7^{\mathrm{a}}$ \\
& Células mononucleares/campo & $40 \pm 9^{\mathrm{c}}$ & $70 \pm 10^{\mathrm{b}}$ & $40 \pm 6^{\mathrm{c}}$ & $50 \pm 8^{\mathrm{b}, \mathrm{c}}$ & $120 \pm 6^{\mathrm{a}}$ \\
& Neovasos/campo & $30 \pm 2^{\mathrm{b}}$ & $80 \pm 10^{\mathrm{a}}$ & $40 \pm 6^{\mathrm{b}}$ & $50 \pm 4^{\mathrm{b}}$ & $50 \pm 10^{\mathrm{b}}$ \\
& Fibroblastos/campo & $10 \pm 6^{\mathrm{a}}$ & $10 \pm 5^{\mathrm{a}}$ & $10 \pm 4^{\mathrm{a}}$ & $10 \pm 3^{\mathrm{a}}$ & $10 \pm 3^{\mathrm{a}}$ \\
& Células mononucleares/campo & $30 \pm 5^{\mathrm{a}}$ & $40 \pm 6^{\mathrm{a}}$ & $40 \pm 5^{\mathrm{a}}$ & $40 \pm 8^{\mathrm{a}}$ & $40 \pm 8^{\mathrm{a}}$ \\
\hline
\end{tabular}




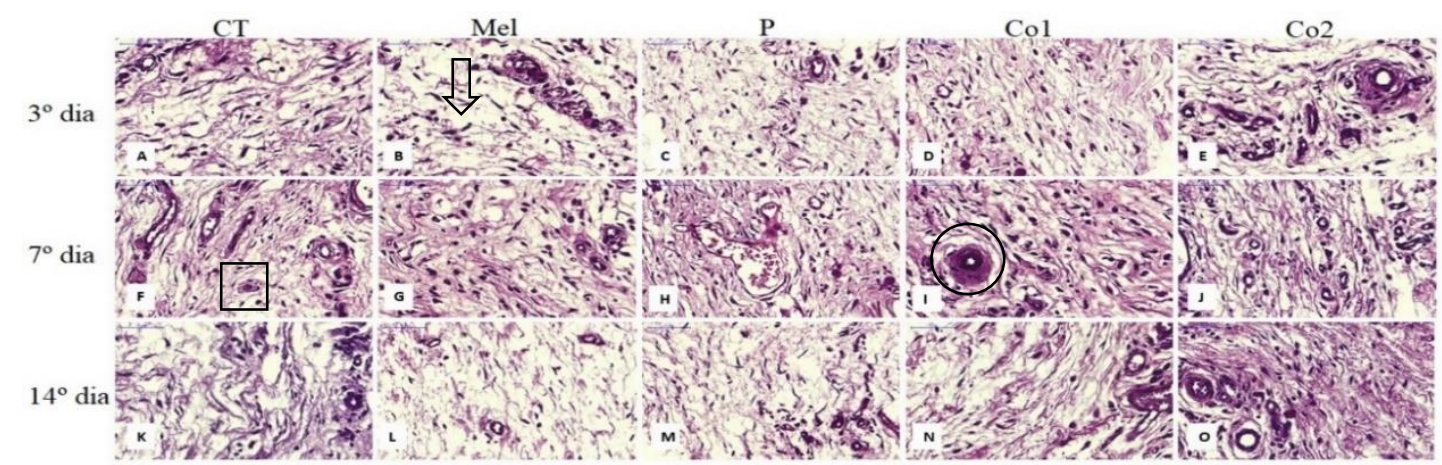

Figura 2. Fotomicrografia de cortes histológicos das feridas cutâneas de ratos Wistar (Rattus norvegicus), corados com hematoxilina-eosina (HE), ao terceiro dia (A-E), sétimo dia (F-J) e $14^{\circ}$ dia (K-O) de experimento dos grupos controle (CT), mel, creme do extrato de Bidens pilosa L. a 10\% (P), pomada comercial alopática - alantoína e óxido de zinco (Co1), pomada comercial homeopática - Bellis perenis + Calendula officinalis + Myristica sebifera (Co2), (400x), demonstrando células mononucleares (quadrado), neovasos (círculo) e fibroblastos (seta).

$\mathrm{Na}$ avaliação das células mononucleares, não foram observadas diferenças entre os grupos tratados com as pomadas $\mathrm{Co} 1$ e $\mathrm{Co} 2$ em relação ao grupo CT; também não se observou diferença entre o grupo CT e o grupo mel, contudo os animais tratados com creme do extrato de $B$. pilosa a $10 \%$ obtiveram menor expressão dessas células. Em relação ao número de neovasos, o grupo tratado com a pomada comercial $2(\mathrm{Co} 2)$ demonstrou aumento significativo, sendo superior aos grupos CT, mel e Co1, não havendo diferença apenas para o grupo P (Tab. 1 e Fig.2). Entretanto, não houve diferença do número de neovasos entre o grupo $\mathrm{P}$ e os grupos CT, mel e Co1. No sétimo dia de tratamento, o grupo $\mathrm{Co} 2$ foi superior ao grupo tratado com creme do extrato de B. pilosa a $10 \%(\mathrm{P})$ com relação ao número de fibroblastos, porém não houve diferenças em relação aos demais grupos. As células mononucleares do grupo $\mathrm{P}$ apresentaram baixa expressividade em relação aos demais grupos, que não demonstraram diferença entre si.

O número de neovasos no grupo $\mathrm{Co} 2$ mostrou-se superior, havendo diferença significativa em relação aos demais grupos. $\mathrm{O}$ grupo mel não demonstrou diferença em relação ao grupo Co1, porém apresentou diferença em comparação com o grupo CT e o grupo P (Tab. 1 e Fig. 2). No $14^{\circ}$ dia, o número de fibroblastos do grupo mel foi maior quando este foi comparado com os demais grupos, que não apresentaram diferença entre si.
$\mathrm{Na}$ contagem de células mononucleares e neovasos na última análise do experimento, não foi observada diferença significativa entre os grupos estudados (Tab. 1 e Fig. 2).

A constituição do colágeno apresentou diferença com relação à espessura das fibras e ao entrelaçamento delas entre os grupos, durante as análises do experimento. O grupo CT apresentou fibras colágenas de fina espessura com pouco entrelaçamento, principalmente no sétimo e $14^{\circ}$ dias do experimento. $\mathrm{O}$ grupo mel demonstrou um espessamento significativo nas fibras de colágeno entre o terceiro e sétimo dias, o qual se manteve até o final do experimento, porém com baixo padrão de entrelaçamento.

O grupo $\mathrm{P}$ apresentou grande quantidade de fibras altamente espessas ao terceiro dia, que se manteve até o sétimo dia, com o mesmo padrão, e diferenciou-se ao término do experimento, mantendo bom entrelaçamento e fibras de espessura mais fina. O grupo Co1 apresentou evolução significativa do terceiro ao sétimo dia em relação à espessura das fibras, chegando ao $14^{\circ}$ dia com fibras mais delgadas e entrelaçadas. $\mathrm{O}$ grupo $\mathrm{Co} 2$ apresentou fibras moderadamente espessadas ao terceiro dia, com bom entrelaçamento. Ao sétimo dia, as fibras tornaram-se mais espessas, com agrupamento distante, e chegaram ao $14^{\circ}$ dia com fibras intensamente entrelaçadas (Fig. 3). 


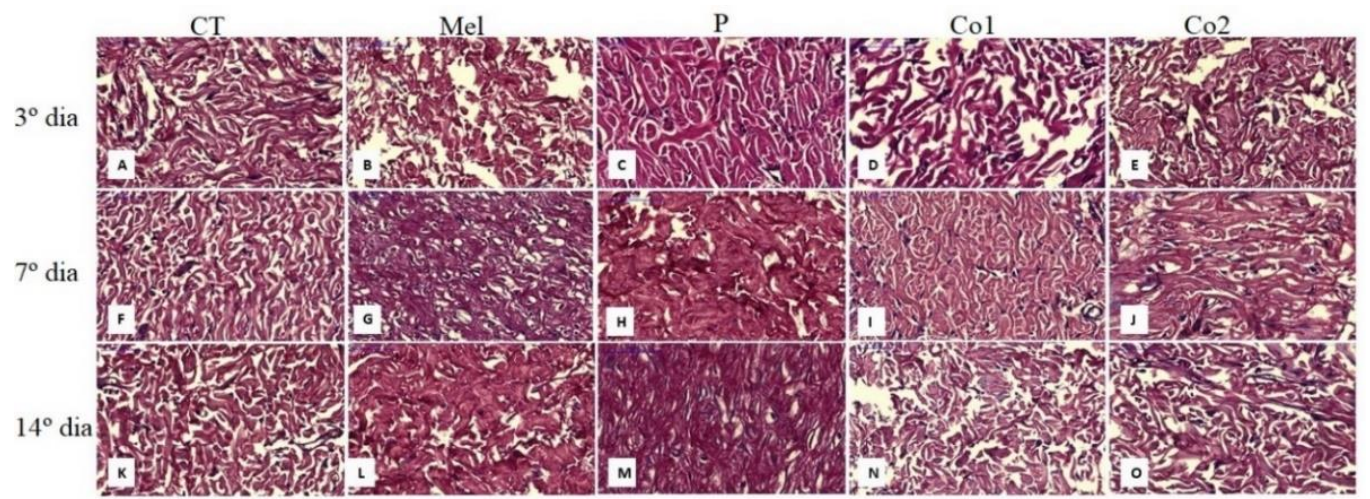

Figura 3. Fotomicrografia de cortes histológicos das feridas cutâneas de ratos Wistar (Rattus norvegicus), coradas com picro sirius red, demonstrando a organização das fibras de colágeno ao terceiro dia (A-E), sétimo dia (F-J) e $14^{\circ}$ dia (K-O) de experimento dos grupos controle (CT), mel, creme do extrato de Bidens pilosa L. a $10 \%(\mathrm{P})$, pomada comercial alopática - alantoína e óxido de zinco (Co1), pomada comercial homeopática - Bellis perenis + Calendula officinalis + Myristica sebifera (Co2), (400x).

\section{DISCUSSÃO}

A evolução clínica na cicatrização das feridas dos animais do grupo $\mathrm{P}$ não demonstrou sinais de infecção aparente e inflamatórios exacerbados durante o experimento, devido à ação antimicrobiana e antifúngica da planta, sendo potencialmente eficiente para inibir o crescimento de Staphylococcus aereus, Staphylococcus epidermides, Escherichia coli, Klebsiella pnuemoniae, Penicillium notatum e Aspergillus niger (Ashafa e Afolayan, 2009). Além disso, apresentou baixa formação de crostas sobre a lesão, favorecendo rápida cicatrização, atingindo fechamento completo no $12^{\circ}$ dia de tratamento.

A lesão tecidual induz a liberação de mediadores inflamatórios resultantes da cascata de ácido araquidônico, como prostaglandinas, leucotrienos e ácido hidroxieicosatetraenoico (Hete) (Trenam et al., 1992). Esses mediadores atraem neutrófilos e macrófagos para a lesão que participam de todas as manifestações clínicas do processo inflamatório/debridamento. Os neutrófilos e macrófagos desempenham papel importante na fagocitose de bactérias, na produção e secreção de proteinases e espécies reativas de oxigênio (ERO) na cicatrização. O aumento de ERO é conhecido como "explosão respiratória", uma vez que a ativação de neutrófilos e macrófagos usa o citocromo NADPH oxidase para reduzir o oxigênio molecular em ânions superóxido (Wientjes e Segal, 1995).
Contudo, esse processo exacerbado traz prejuízo ao tecido e, no presente estudo, observou-se menor número de células mononucleares nas feridas dos animais do grupo $\mathrm{P}$ em relação aos demais grupos estudados. Esse achado pode ser pela ação dos flavonoides de B. pilosa (Gilbert e Favoreto, 2013), capazes de amenizar a ação da ciclo-oxigenase (COX) e da 5-lipoxigenase (Vieira, 2015), o que demonstra que o creme do extrato de B. pilosa a $10 \%$ teve ação antiinflamatória e manteve o processo de debridamento, observado clinicamente pelo menor depósito de crostas nas feridas.

Estudo realizado por Kohda et al. (2013) demonstrou a atividade antioxidante do extrato de B. pilosa, que fomentou aumento na expressão de fatores antioxidantes, diminuindo a produção de espécies reativas de oxigênio em células estimuladas com fator de necrose tumoral alfa (TNF- $\alpha$, tumor necrosis factor alpha), e promoveu a regulação na produção de óxido nítrico (NO, nitric oxid). Essa ação pode ser impulsionada pelos flavonoides presentes na planta, amenizando, assim, o processo inflamatório.

O creme do extrato aquoso a $10 \%$ de B. pilosa, no presente estudo, mostrou eficiência significativa no auxílio à reparação tecidual de feridas cutâneas, apresentando quantidade de neovasos entre o grupo $\mathrm{Co} 2$ e os demais grupos estudados. $\mathrm{O}$ efeito anti-inflamatório de $B$. pilosa nos animais tratados com o creme a $10 \%$ do extrato da planta reduziu o tempo de cicatrização em relação ao CT. 
Além disso, a redução do $\mathrm{pH}$ e da tensão de oxigênio na ferida cutânea induz a angiogênese (Diegelmann et al., 1981; Knighton et al., 1981). Portanto, a aplicação tópica do creme da $B$. pilosa na ferida manteve a integridade das paredes dos vasos sanguíneos, favoreceu a angiogênese na fase inflamatória da cicatrização, o que melhorou o suprimento sanguíneo para a ferida e favoreceu a formação de uma matriz provisória para a fase de proliferação (formação do tecido de granulação) no processo de cicatrização.

$\mathrm{Na}$ análise dos aspectos histológicos das fibras colágenas, as feridas dos animais do grupo $\mathrm{P}$ apresentaram boa conformação durante $\mathrm{O}$ experimento, progredindo na fase de proliferação da cicatrização, as quais deixaram de apresentar grande volume ao sétimo dia de experimento, para adquirirem um padrão de entrelaçamento ao $14^{\circ}$ dia, porém em menor quantidade. Essa evolução na conformação do colágeno entre o sétimo e $14^{\circ}$ dias refere-se à fase de maturação, em que a taxa de síntese diminui e, assim, aumenta-se o número de ligações cruzadas entre as fibras colágenas, o que promove aumento da força tênsil e evita ocorrência de cicatrizes proeminentes (Tazima et al., 2008).

Estudos demonstraram que o mel no processo de reparação tecidual promove quimiotaxia de macrófagos, proliferação de fibroblastos $\mathrm{e}$ atividade antibacteriana, devido à liberação do peróxido de hidrogênio a partir da glicose e presença de inibina, além da sua capacidade higroscópica e do pH baixo (Asaristizabal et al., 2015; Marques et al., 2015). As feridas cutâneas dos animais tratados com mel, no presente estudo, apresentaram, nos primeiros dias, rápida contração da ferida e boa constituição celular na análise histológica. Porém, não apresentaram resultado satisfatório ao final do experimento em relação ao tempo de cicatrização e à aparência da lesão, com um acúmulo excessivo de crostas, prejudicando o processo final da cicatrização.

Observou-se também que a conformação das fibras colágenas não diferiu entre o sétimo e $14^{\circ}$ dias de experimento; elas apresentavam-se espessas, com baixo grau de entrelaçamento. Para que ocorra uma boa cicatrização, a síntese de colágeno, mediada por fibroblastos na fase final da cicatrização, deve ser diminuída pela ação da enzima colagenase, a qual diminui a espessura das fibras para que haja maior entrelaçamento das fibras, garantindo resistência e melhor aparência na cicatriz (Tazima et al., 2008). Marques et al. (2015) também consideraram o mel uma boa opção cicatrizante, com custo reduzido em relação aos produtos comerciais, porém enfatizam a necessidade de novos estudos devido aos poucos relatos que comprovem a segurança na utilização do produto.

El-Kesed et al. (2017) utilizaram, em seu estudo, hidrogel à base de mel na cicatrização de feridas causadas por queimaduras em ratos. O tratamento com hidrogel demonstrou bom resultado clínico, superando os produtos comerciais comparados. No presente estudo, utilizou-se o mel in natura, que demonstrou boa resposta inicial, porém predispôs a um acúmulo excessivo de crostas sobre a lesão, prejudicando o processo final da cicatrização.

Os animais do grupo Co1 apresentaram aumento no número de células mononucleares no terceiro dia de tratamento e demonstraram boa conformação de fibras colágenas, obtendo cicatrização completa no $13^{\circ}$ dia de tratamento. Segundo Laureano e Rodrigues (2011), os macrófagos são considerados as células mais importantes da fase inflamatória, pois, além de fagocitarem agentes patogênicos e debris celulares, são também responsáveis pela liberação de diversos fatores de crescimento, como fator de crescimento derivado de plaquetas (PDGF, platelet-derived growth factor), fator de crescimento tumoral alfa (TGF- $\alpha$, tumor growth factor alpha), fator de crescimento tumoral beta (TGF- $\beta$, tumor growth factor beta), fator de crescimento dos fibroblastos (FGF, fibroblast growth factor) e fator de crescimento endotelial vascular (VEGF, vascular endothelial growth factor), o que proporciona evolução da cicatrização para a fase proliferativa e desenvolvimento do tecido de granulação. Araújo et al. (2010) relataram, em seus estudos obtidos por meio de análises histológicas, que o mecanismo de cicatrização induzido pela alantoína ocorre via controle da resposta inflamatória, a qual estimula a quimiotaxia de fibroblastos e macrófagos de maneira mais intensa e rápida que os grupos controle.

O grupo $\mathrm{Co} 2$, no presente estudo, promoveu cicatrização rápida, obtendo fechamento completo da lesão no $11^{\circ}$ dia de tratamento, com boa aparência estética e baixo acúmulo de crostas 
sobre a ferida. Apresentou grande número de neovasos e fibroblastos na avaliação histológica, acarretando boa conformação das fibras colágenas, quando comparado com o grupo CT. Fernandes et al. (2014) observaram, em seu experimento, que animais tratados com a pomada homeopática (Bellis perenis + Calendula officinalis + Myristica sebifera) apresentaram uma cicatrização mais rápida que os animais do grupo controle, com pouca formação de crostas, o que promoveu melhor aparência cicatricial. Parente et al. (2011) evidenciaram, em seus estudos, o efeito angiogênico de Calendula officinalis, que ocorre devido à presença de triterpenos e esteroides em sua composição e não pela inflamação, conforme ocorre em outros produtos cicatrizantes.

Esse potencial angiogênico e não inflamatório permitiu uma cicatrização mais limpa e com menor acúmulo de crostas nos animais do grupo $\mathrm{Co} 2$ no presente estudo. No experimento realizado por Karakas et al. (2011), foi observado histologicamente que os animais tratados com o extrato de Bellis perenis apresentaram fibras de colágeno mais agrupadas e entrelaçadas, quando comparados aos animais do grupo controle, o que garantiu melhor aparência estética no local da lesão, assim como ocorreu no grupo $\mathrm{Co} 2$ no presente estudo.

\section{CONCLUSÃO}

De acordo com o modelo experimental proposto no presente estudo, todos os tratamentos demonstraram potencial cicatrizante, com exceção do mel. A aplicação tópica do creme do extrato de Bidens pilosa L. a $10 \%$ apresentou melhor perfil anti-inflamatório; a pomada alopática apresentou boa aderência à superfície da lesão e a pomada homeopática, grande potencial angiogênico, com menor tempo de cicatrização.

\section{AGRADECIMENTOS}

À Unifenas, à Capes e ao IFSULDEMINAS, por fornecer apoio financeiro, por conceder bolsa de estudo e por ceder a estrutura necessária para o desenvolvimento do experimento, respectivamente.

\section{REFERÊNCIAS}

ALVES, R.M.O; CARVALHO, C.A.L.; SOUZA, B.A. et al. Características de mel de Melipona mandacaia Smith (Hymenoptera: Apidae). Ciênc. Tecnol. Aliment., v.25, p.644650, 2005.

ARAÚJO, E.C. A cicatrização de feridas com o uso de produtos naturais. Rev. Enfermag. UFPE OnLine, v.10, p.1-2, 2016.

ARAÚJO, L.U.; GRABE-GUIMARÃES, A.; MOSQUEIRA, V.C. et al. Profile of wound healing process induced by allantoin. Acta Cir. Bras., v.25, p.460-466, 2010.

ASARISTIZABAL, A.S.; HAYASHI, A.M.; MATERA, J.M. Use of organic honey for the topic treatment of a third-degree skin burn in a dog - case report. Rev. Educ. Cont. Med. Vet. Zootec. CRMV SP., v.14, p.12-17, 2015.

ASHAFA, A.O.T.; AFOLAYAN, A.J. Screening the root extracts from Bidens pilosa $L$. var. radiata (Asteraceae) for antimicrobial potential. J. Med. Plants Res., v.3, p.568-572, 2009.

BACH, F.T.; SILVA, C.A.T. Efeito alelopático de extrato aquoso de boldo e picão preto sobre a germinação e desenvolvimento de plantas de alface. Cultivando Saber, v.3, p.190-198, 2010.

COELHO, J.M.; ANTONIOLLI, A.B.; SILVANUNES, D. et al. Efeito da sulfadiazina de prata, extrato de ipê-roxo e extrato de barbatimão na cicatrização de feridas cutâneas em ratos. Rev. Col. Bras. Cir., v.37, p.45-51, 2010.

DIEGELMANN, R.F.; COHEN, I.K.; KAPLAN, A.M. O papel dos macrófagos no reparo de feridas: uma revisão. Cir. Plást. Reconstr., v.68, p.107-113, 1981.

EL-KESED, R.F.; AMER, R.I.; ATTIA, D. et al. Honey-based hydrogel: in vitro and comparative in vivo evaluation for burn wound healing. Sci. Rep., v.7, p.1-9, 2017.

FERNANDES, E.S.; SANTOS, R.A.; OLIEIRA, B.S. et al. Comparative analysis of the use of CMR pomade (Bellis perenis + Calendula officinalis + Myristica sebifera) and Physiological solution in tissue scarring of rats (Rathus norvegicus). Sci. Electr. Archs., v.7, p.103-107, 2014. 
FOTSO, A.F.; LONGO, F.; DJOMENI, P.D.D. et al. Atividades analgésicas e anti-inflamatórias da fração acetato de etila de Bidens pilosa (Asteracea). Inflammopharmacol, v.22, p.105114, 2014.

GEISSBERGER, P.; SÉQUIN, U. Constituents of Bidens pilosa $\mathrm{L} .:$ do the components found so far explain the use of this plant in traditional medicine? Acta Trop., v.48, p.251-261, 1991.

GILBERT, B.; FAVORETO, R. Bidens pilosa L. Asteraceae (Compositae; subfamília Heliantheae). Rev. Fitos, v.8, p.53-62, 2013.

HAIDA, K.S.; PARZIANELLO, L.; WERNER, S. et al. Avaliação in vitro da atividade antimicrobiana de oito espécies de plantas medicinais. Arq. Ciênc. Saúde Unipar, v.11, p.185-192, 2007.

HERNANDES, L.; PEREIRA, L.M.S.; PALAZZO, F. et al. Wound healing evaluation of ointment from Stryphnodendron Adstringens (barbatimão) in rat skin. Braz. J. Pharm. Sci., v.46, p.431-436, 2010.

JUNQUEIRA, L.C.; CARNEIRO, J. Histologia básica: texto e atlas. 10.ed. Rio de Janeiro: Guanabara Koogan, 2004. 488p.

KARAKAS, F.P.; KARAKAS, A.; BORAN, C. et al. The evaluation of topical administration of Bellis perennis fraction on circular excision wound healing in Wistar albino rats. Pharma. Biol., v.50, p.1031-1037, 2011.

KNIGHTON, D.R.; SILVER, I.; HUNT, T.K. Regulamentação do efeito da angiogênese na cicatrização de feridas dos gradientes de oxigênio e oxigênio inspirado. Surgery, v.90, p.262-270, 1981.

KOHDA, F.; TAKAHARA, M.; HACHIYA, A. et al. Decrease of reactive oxygen species and reciprocal increase of nitric oxide in human dermal endothelial cells by Bidens pilosa extract: a possible explanation of its beneficial effect on livedo vasculopathy. J. Dermatol. Sci., v.72, p.7577, 2013.

LAUREANO, A.; RODRIGUES, A.M. Cicatrização de feridas: educação médica contínua. Rev. SPDV, v.69, p.355-363, 2011.

MANDELBAUM, S.H.; DI SANTIS, E.P.; MANDELBAUM, M.H.S. Cicatrização: conceitos atuais e recursos auxilares - Parte I. Anais Bras. Dermatol., v.78, p.393-408, 2003.
MARQUES, A.D.B.; SANTOS, L.M.D.; MAGALHÃES, P.H. et al. O uso do mel no tratamento de feridas de difícil cicatrização: revisão sistemática. Rev. Prev. Infec. Saúde, v.1, p.42-51, 2015.

MORESKI, D.A.B.; LEITE-MELLO, E.V.S.; BUENO, F.G. Ação cicatrizante de plantas medicinais: um estudo de revisão. Arq. Ciênc. Saúde UNIPAR, v.22, p.63-69, 2018.

OLIVEIRA, F.Q; ANDRADE-NETO, V.; KRETTLI, A.U. et al. New evidences of antimalarial activity of Bidens pilosa roots extract correlated with polyacetylene and flavonoids. $J$. Ethnopharmacol., v.93, p.39-42, 2004.

OLIVEIRA, I.V.P.M.; DIAS, R.V.C. Cicatrização de feridas: fases e fatores de influência. Acta Vet. Bras., v.6, p.267-271, 2012.

PARENTE, L.M.L.; ANDRADE, M.A.M.; BRITO, L.A.B. et al. Angiogenic activity of Calendula officinalis L. flowers in rats. Acta Cir. Bras., v.26, p.19-24, 2011.

TAGAMI, O.K.; GASPARINI, M.D.G.; SCHWAN-ESTRADA, K.R.F. et al. Fungitoxidade de bidens pilosa, thymus vulgaris, lippia alba e rosmarinus officinalis no desenvolvimento in vitro de fungos fitopatogênicos. Ciênc. Agrár., v.30, p.285-294, 2009.

TAZIMA, M.F.G.S.; VICENTE, Y.; MORIYA, T. Biologia de feridas e cicatrização. Simp. Fundam. Clín. Cir., v.41, p.259-264, 2008.

TRENAM, C.W.; DABBAGH, A.J.; BLAKE, D.R.; MORRIS, C.J. O papel do ferro em um modelo agudo de inflamação da pele induzida por espécies reativas de oxigênio. Br. J. Dermatol., v.126, p.250-256, 1992.

VIEIRA, G.T. Avaliação do efeito cicatrizante de Ingasubnuda e Pseudopiptadenia contorta em feridas cirúrgicas em coelhos. 2015. 123f. Tese (Doutorado em Bioquímica Agrícola) Universidade Federal de Viçosa, Viçosa, MG.

WIENTJES, F.B.; SEGAL, A.W. NADPH oxidase e a explosão respiratória. Semin. Biol. Cel., v.6, p.357-365, 1995. 